GANIT J. Bangladesh Math. Soc. (ISSN 1606-3694) 34 (2014) 27-33

\title{
COMMUTATIVELY OF PRIME AND SEMIPRIME Г-RINGS WITH SYMMETRIC BI-DERIVATIONS
}

\author{
Kalyan Kumar Dey ${ }^{1}$ and Akhil Chandra Paul ${ }^{2}$ \\ ${ }^{1,2}$ Department of Mathematics, University of Rajshahi, Rajshahi-6205, Bangladesh \\ ${ }^{1}$ kkdmath@yahoo.com; ${ }^{2}$ acpaulrubd_math@yahoo.com
}

Received 28.05.2013

Accepted 07.12.2014

\begin{abstract}
Let $M$ be a $\Gamma$-ring and let $D: M \times M \rightarrow M$ be a symmetric bi-derivation with the trace $d$ : $M \rightarrow M$ denoted by $d(x)=D(x, x)$ for all $x \in M$. The objective of this paper is to prove some results concerning symmetric bi-derivation on prime and semiprime $\Gamma$-rings. If $M$ is a 2-torsion free prime $\Gamma$-ring and $D \neq 0$ be a symmetric bi-derivation with the trace $d$ having the property $d(x) \alpha x-x \alpha d(x)=0$ for all $x \in M$ and $\alpha \in \Gamma$, then $M$ is commutative. We also prove another result in $\Gamma$-rings setting analogous to that of Posner for prime rings.
\end{abstract}

Keywords: $\Gamma$-ring, derivation, bi-derivation, commutativity

\section{Introduction and Preliminaries}

The concept of a $\Gamma$-ring was first introduced by Nobuswa [5], and afterwards it was generalized by Barnes [1] in more natural sense. Maksa [14] worked on the trace of symmetric bi-derivation on classical rings theories and developed some fruitful results concerning bi-derivations. Vukman [10] proved some results relating symmetric biderivations on prime and semiprime rings. Ozturk, Sapanci, Soyturk and Kim [7] worked on the trace of symmetric bi-derivations in $\Gamma$-rings and extended some results of Vukman $[10]$ to ideals of prime and semipime $\Gamma$-rings.

In this paper, we extend some results of Vukman [10] to prime and semiprime $\Gamma$-rings. Our results are quiet different from the results obtained in [9].

Let $M$ and $\Gamma$ be additive abelian groups. If there exists a mapping $(x, \alpha, y) \rightarrow x \alpha y$ of $M \times \Gamma \times M \rightarrow M$ which satisfies the conditions:

(i) $x \alpha y \in M$,

(ii) $(x+y) \alpha z=x \alpha z+y \alpha z, x(\alpha+\beta) y=x \alpha y+x \beta y, x \alpha(y+z)=x \alpha y+x \alpha z$,

(iii) $(x \alpha y) \beta z=x \alpha(y \beta z)$ for all $x, y, z \in M$ and $\alpha, \beta \in \Gamma$,

then $M$ is called a $\Gamma$-ring in the sense of Barnes [1]. Throughout this paper $M$ denotes a $\Gamma$-ring with center $Z(M)$. For any $x, y \in M, \alpha \in \Gamma$, the symbol $[x, y]_{\alpha}$ (resp. $\langle x, y\rangle_{\alpha}$ ) will denote the commutator $x \alpha y-y \alpha x$ (resp. the anti-commutator $x \alpha y+y \alpha x$ ). A $\Gamma$-ring $M$ is called commutative if $[x, y]_{\alpha}=0$ for all $x, y \in M, \alpha \in \Gamma$. We know that

$$
[x \beta y, z]_{\alpha}=[x, z]_{\alpha} \beta y+x \beta[y, z]_{\alpha}+x[\beta, \alpha]_{z} y
$$


and

$$
[x, y \beta z]_{\alpha}=y \beta[x, z]_{\alpha}+[x, y]_{\alpha} \beta z+y[\beta, \alpha]_{x} z .
$$

We make the assumption (*) $x \beta z \alpha y=x \alpha z \beta y$ for all $x, y, z \in M, \alpha, \beta \in \Gamma$. Using this assumption the basic commutator identities reduce to

$$
\begin{aligned}
& {[x \beta y, z]_{\alpha}=[x, z]_{\alpha} \beta y+x \beta[y, z]_{\alpha}} \\
& {[x, y \beta z]_{\alpha}=y \beta[x, z]_{\alpha}+[x, y]_{\alpha} \beta z .}
\end{aligned}
$$

Recall that a $\Gamma$-ring $M$ is prime if $x \Gamma M \Gamma y=0$ implies that $x=0$ or $y=0$, and is semiprime if $x \Gamma M \Gamma x=0$ implies $x=0$. An additive mapping $d: M \rightarrow M$ is called a derivation if $d(x \alpha y)=d(x) \alpha y+x \alpha d(y)$ holds for all $x, y \in M, \alpha \in \Gamma$. A derivation $d$ is inner if there exists $a \in M$ such that $d(x)=[a, x]_{\alpha}$ holds for all $x \in M, \alpha \in \Gamma$. The mapping $B: M \times M \rightarrow M$ is said to by symmetric if $B(x, y)=B(y, x)$ holds for all $x, y \in M$. A mapping $f: M \rightarrow M$ defined by $f(x)=B(x, x)$, where $B: M \times M \rightarrow M$ is a symmetric mapping, is called the trace of $B$. In case $B: M \times M \rightarrow M$ is a symmetric mapping which is also bi-additive (i.e. additive in both arguments), the trace of $B$ satisfies the relation $f(x+y)=f(x)+f(y)+2 B(x, y)$, for all $x, y \in M$. We shall use also the fact that the trace of a symmetric bi-additive mapping is an even function. A symmetric bi-additive mapping $D: M \times M \rightarrow M$ is called a symmetric biderivation if $D(x \alpha y, z)=D(x, z) \alpha y+x \alpha D(y, z)$ is fulfilled for all $x, y, z \in M, \alpha \in \Gamma$. Obviously, in this case also the relation $D(x, y \alpha z)=D(x, y) \alpha z+y \alpha D(x, z)$ for all $x, y$, $z \in M, \alpha \in \Gamma$, holds. A mapping $f: M \rightarrow M$ is said to be commuting on $M$ if $[f(x), x]_{\alpha}=0$ holds for all $x \in M, \alpha \in \Gamma$. A mapping $f: M \rightarrow M$ is centralizing on $M$ if $[f(x), x]_{\alpha} \in Z(M)$ holds for all $x \in M, \alpha \in \Gamma$.

\section{Bi-derivations on $\Gamma$-rings}

We shall need the following well-known and frequently used lemmas.

Lemma 2.1. ([2, Lemma 3.2]) Let $d: M \rightarrow M$ be a derivation, where $M$ is a prime $\Gamma$-ring. Suppose that either (i) $a \Gamma d(x)=0$, for all $x \in M$ or (ii) $d(x) \Gamma a=0$, for all $x \in M$ holds. Then we have (i) $a=0$ or (ii) $d=0$.

Lemma 2.2. ([7, Lemma 3]) Let $M$ be a 2-torsion free prime $\Gamma$-ring and let $a, b \in M$ be fixed elements. If $a \alpha x \beta b+b \alpha \times \beta a=0$ is fulfilled for all $x \in M, \alpha, \beta \in \Gamma$, then $a=0$ or $b=0$.

We start our investigation of symmetric bi-derivations with the following results.

Theorem 2.3. Let $M$ be a 2-torsion free prime $\Gamma$-ring satisfying the condition (*). Let $D: M \times M \rightarrow M$ and $d$ be a symmetric bi-derivation and the trace of $D$, respectively. Suppose that $d$ is commuting on $M$, then $M$ is commutative or $D=0$.

Proof. We have

$$
[d(x), x]_{\alpha}=0 \text {, for all } x \in M, \alpha \in \Gamma \text {. }
$$

The linearization of (1) gives us $[d(x)+d(y)+2 D(x, y), x+y]_{\alpha}=0$,

which leads to

$$
[d(x), y]_{\alpha}+[d(y), x]_{\alpha}+2[D(x, y), x]_{\alpha}+2[D(x, y), y]_{\alpha}=0 \text { for all } x, y \in M, \alpha \in \Gamma .
$$


Substituting $-x$ for $x$ in the relation above, we arrive at

$$
[d(x), y]_{\alpha}-[d(y), x]_{\alpha}+2[D(x, y), x]_{\alpha}-2[D(x, y), y]_{\alpha}=0 \text { for all } x, y \in M, \alpha \in \Gamma .
$$

From (2) and (3) we obtain

$$
[d(x), y]_{\alpha}+2[D(x, y), x]_{\alpha}=0 \text { for all } x, y \in M, \alpha \in \Gamma .
$$

Replacing $y$ in (4) by $x \beta y$. Then by using the condition $(*)$,

$$
\begin{aligned}
0 & =[d(x), x \beta y]_{\alpha}+2[d(x) \beta y+x \beta D(x, y), x]_{\alpha} \\
& =x \beta[d(x), y]_{\alpha}+2 d(x) \beta[y, x]_{\alpha}+2 x \beta[D(x, y), x]_{\alpha}
\end{aligned}
$$

which, according to (4), implies

$$
d(x) \beta[x, y]_{\alpha}=0 \text { for all } x, y \in M, \alpha, \beta \in \Gamma .
$$

From the relation (5) and Lemma 2.1 one can conclude that $d(x)=0$ or $[x, y]_{\alpha}=0$ for all $x, y \in M, \alpha \in \Gamma$. If $[x, y]_{\alpha}=0$, then $M$ is commutative. On the other hand, for any $x \notin Z(M)$, we have $[\mathrm{x}, \mathrm{y}]_{\alpha} \neq 0$. Therefore $d(x)=0$ (note that for any fixed $x \in M, \alpha \in \Gamma$, a mapping $y \rightarrow[x, y]_{\alpha}$ is a derivation). Let $x \in Z(M), y \notin Z(M)$. Then $x+y \notin Z(M)$ and $x-y \notin Z(M)$. Thus $0=d(x+y)=d(\mathrm{x})+2 D(x, y)$ and $0=d(x)-2 D(x, y)$. From these two relations, we have $4 D(x, y)=0$. By the 2-torsion freeness of $M$, we have

$$
D(x, y)=0 \text { for all } x, y \in M \text {. The proof of the theorem is complete. }
$$

Theorem 2.4. Let $M$ be a 2 and 3-torsion free prime $\Gamma$-ring satisfying the condition (*). Let $D: M \times M \rightarrow M$ and $d$ be a symmetric bi-derivation and the trace of $D$, respectively. Suppose that $d$ is centralizing on $M$, then $M$ is commutative or $D=0$.

Proof We have

$$
[d(x), x]_{\alpha} \in Z(M) \text { for all } x \in M, \alpha \in \Gamma .
$$

By linearization we obtain

$$
\begin{gathered}
{[d(x)+d(y)+2 D(x, y), x+y]_{\alpha} \in Z(M)} \\
\Rightarrow[d(y), x]_{\alpha}+[d(x), y]_{\alpha}+2[D(x, y), y]_{\alpha}+2[D(x, y), x]_{\alpha} \in Z(M) \text { for all } x, y \in M, \alpha \in \Gamma .
\end{gathered}
$$

since (6) holds. Replacing $x$ in the relation (7) by $-x$, we obtain

$-[d(y), x]_{\alpha}+[d(x), y]_{\alpha}-2[D(x, y), y]_{\alpha}+2[D(x, y), x]_{\alpha} \in Z(M)$ for all $x, y \in M, \alpha \in \Gamma$. (8)

Now (7) and (8) give us

$$
[d(x), y]_{\alpha}+2[D(x, y), x]_{\alpha} \in Z(M) \text { for all } x, y \in M, \alpha \in \Gamma \text {. }
$$

Replacing $y$ in (9) by $x \beta x$, we get

$$
\begin{aligned}
& {[d(x), x \beta x]_{\alpha}+2[d(x) \beta x+x \beta d(x), x]_{\alpha} \in Z(M)} \\
& \Rightarrow[d(x), x]_{\alpha} \beta x+x \beta[d(x), x]_{\alpha}+2[d(x), x]_{\alpha} \beta x+2 x \beta[d(x), x]_{\alpha} \in Z(M) \\
& \Rightarrow 6[d(x), x]_{\alpha} \beta x \in Z(M) \text { for all } x, y \in M, \alpha, \beta \in \Gamma .
\end{aligned}
$$


Using (10), (6) and the assumptions that $M$ is 2 and 3-torsion free, we obtain

$$
[d(x), x]_{\alpha} \beta[x, y]_{\alpha}=0 \text { for all } x, y \in M, \alpha, \beta \in \Gamma .
$$

Now, the relation above makes it possible to conclude, using the same arguments as in the proof of Theorem 2.3, that for any $x \notin Z(M)$ we have $[d(x), x]_{\alpha}=0$.

In view of Theorem 2.3, the proof is complete.

Theorem 2.5. Let $M$ be a 2-torsion free prime $\Gamma$-ring satisfying the condition $\left({ }^{*}\right)$. Suppose there exist symmetric bi-derivations $D_{1}: M \times M \rightarrow M$ and $D_{2}: M \times M \rightarrow M$, such that $D_{1}\left(d_{2}(x), x\right)=0$ holds for all $x \in M$, where $d_{2}$ denotes the trace of $D_{2}$.

Then $D_{1}=0$ or $D_{2}=0$.

Proof. By linearization of the relation

$$
D_{1}\left(d_{2}(x), x\right)=0 \text { for all } x \in M .
$$

we obtain according to (11),

$$
\begin{aligned}
& D_{1}\left(d_{2}(x)+d_{2}(y)+2 D_{2}(x, y), x+y\right)=0 \\
\Rightarrow & D_{1}\left(d_{2}(y), x\right)+2 D_{1}\left(D_{2}(x, y), x\right)+D_{1}\left(d_{2}(x), y\right)+2 D_{1}\left(D_{2}(x, y), y\right)=0 \text { for all } x, y \in M .
\end{aligned}
$$

Replacing $x$ by $-x$ and comparing this new equation with the preceding equation we get

$$
D_{1}\left(d_{2}(x), y\right)+2 D_{1}\left(D_{2}(x, y), x\right)=0 \text { for all } x, y \in M .
$$

Let us replace $y$ by $x \alpha y$ in (12). Then

$$
\begin{aligned}
0 & =D_{1}\left(d_{2}(x), x \alpha y\right)+2 D_{1}\left(D_{2}(x, x \alpha y), x\right) \\
& =D_{1}\left(d_{2}(x), x\right) \alpha y+x \alpha D_{1}\left(d_{2}(x), y\right)+2 D_{1}\left(d_{2}(x) \alpha y+x \alpha D_{2}(x, y), x\right) \\
& =x \alpha D_{1}\left(d_{2}(x), y\right)+2 D_{1}\left(d_{2}(x), x\right) \alpha y+2 d_{2}(x) \alpha D_{1}(y, x)+2 d_{1}(x) \alpha D_{2}(x, y)+2 x \alpha D_{1}\left(D_{2}(x, y), x\right) \\
& =x \alpha D_{1}\left(d_{2}(x), y\right)+2 x \alpha D_{1}\left(D_{2}(x, y), x\right)+2 d_{2}(x) \alpha D_{1}(x, y)+2 d_{1}(x) \alpha D_{2}(x, y) \\
& =2 d_{2}(x) \alpha D_{1}(x, y)+2 d_{1}(x) \alpha D_{2}(x, y) .
\end{aligned}
$$

In the above calculation we used (11) and (12). Thus we have

$$
d_{2}(x) \alpha D_{1}(x, y)+d_{1}(x) \alpha D_{2}(x, y)=0 \text { for all } x, y \in M, \alpha \in \Gamma .
$$

Let us replace $y$ in (13) by $y \beta x$. We get

$$
\begin{aligned}
0 & =d_{2}(x) \alpha D_{1}(y \beta x, x)+d_{1}(x) \alpha D_{2}(y \beta x, x) \\
& =d_{2}(x) \alpha\left(D_{1}(y, x) \beta x+y \beta d_{1}(x)\right)+d_{1}(x) \alpha\left(D_{2}(y, x) \beta x+y \beta d_{2}(x)\right) \\
& =\left(d_{2}(x) \alpha D_{1}(x, y)+d_{1}(x) \alpha D_{2}(x, y)\right) \beta x+d_{1}(x) \alpha y \beta d_{2}(x)+d_{2}(x) \alpha y \beta d_{1}(\mathrm{x}) \\
& =d_{1}(x) \alpha y \beta d_{2}(x)+d_{2}(x) \alpha y \beta d_{1}(x) .
\end{aligned}
$$

Thus, we have

$$
d_{1}(x) \alpha y \beta d_{2}(x)+d_{2}(x) \alpha y \beta d_{2}(x)=0 \text { for all } x, y \in M, \alpha, \beta \in \Gamma .
$$


Let us assume that $d_{1}$ and $d_{2}$ are both different from zero. In other words there exist elements $x_{1}, x_{2} \in M$ such that $d_{1}\left(x_{1}\right) \neq 0$ and $d_{2}\left(x_{2}\right) \neq 0$. From (14) and Lemma 2.2, it follows that $d_{1}\left(x_{2}\right)=d_{2}\left(x_{2}\right)=0$. Since $d_{1}\left(x_{2}\right)=0$, the relation (13) reduces to $d_{2}\left(x_{2}\right) \alpha D_{1}\left(x_{2}\right.$, $y)=0$. Using this relation and Lemma 2.1, we obtain that $D_{1}\left(x_{2}, y\right)=0$ holds for all $y \in M$ since $d_{2}\left(x_{2}\right) \neq 0$ (recall that a mapping $y \rightarrow D_{1}\left(x_{2}, y\right)$ is a derivation). In particular we have $D_{1}\left(x_{2}, x_{1}\right)=0$. Similarly, we obtain $D_{2}\left(x_{1}, x_{2}\right)=0$ holds as well. Let us write $y$ for $x_{1}+x_{2}$. Then $d_{1}(y)=d_{1}\left(x_{1}+x_{2}\right)=d_{1}\left(x_{1}\right)+d_{1}\left(x_{2}\right)+2 D_{1}\left(x_{1}, x_{2}\right)=d_{1}\left(x_{1}\right) \neq 0$. Similarly, we obtain $d_{2}(y)$ $\neq 0$. But $d_{1}(y)$ and $d_{2}(y)$ cannot be both different from zero according to (14) and Lemma 2.2. Therefore we have proved that $d_{1}=0$ or $d_{2}=0$ which is the assertion of the theorem.

In case $D_{1}=D_{2}$ Theorem 2.5 can be proved for semiprime $\Gamma$-rings.

Theorem 2.6. Let $M$ be a 2-torsion free semiprime $\Gamma$-ring. Suppose there exists such a symmetric bi-derivation $D: M \times M \rightarrow M$ that $D(d(x), x)=0$ holds for all $x \in M$, where $d$ denotes the trace of $D$. Then $D=0$.

Proof. In this case (14) reduces to $d(x) \alpha y \beta d(x)=0$ for $x, y \in M, \alpha, \beta \in \Gamma$, which implies that $d(x)=0$ for all $x \in M$, by semiprimeness of Posner [10] has proved a result which states that in case $M$ is a 2-torsion free prime $\Gamma$-ring and $D_{1}, D_{2}$ are nonzero derivations on $M$, then the mapping $x \rightarrow D_{1}\left(D_{2}(x)\right)$ cannot be a derivation.

The result below was motivated by Posner's result mentioned above.

Theorem 2.7. Let $M$ be a 2 and 3-torsion free prime $\Gamma$-ring satisfying the condition (*). Let $D_{1}: M \times M \rightarrow M$ and $D_{2}: M \times M \rightarrow M$ be symmetric bi-derivations. Suppose further that there exists a symmetric bi-additive mapping $B: M \times M \rightarrow M$ such that $d_{1}\left(d_{2}(x)\right)=f(x)$ holds for all $x \in M$, where $d_{1}$ and $d_{2}$ are the traces of $D_{1}$ and $D_{2}$, respectively, and $f$ is the trace of $B$. Then $D_{1}=0$ or $D_{2}=0$.

Proof. The linearization of the relation

$$
d_{1}\left(d_{2}(x)\right)=f(x) \text { for all } x \in M .
$$

gives us

$$
d_{1}\left(d_{2}(x)+d_{2}(y)+2 D_{1}(x, y)\right)=f(x)+f(y)+2 B(x, y)
$$

and

$d_{1}\left(d_{2}(x)\right)+d_{1}\left(d_{2}(y)\right)+4 d_{1}\left(D_{2}(x, y)\right)+2 D_{1}\left(d_{2}(x), d_{2}(y)\right)+4 D_{1}\left(d_{2}(x), D_{2}(x, y)\right)+4 D_{1}\left(d_{2}(y)\right.$, $\left.D_{2}(x, y)\right)=f(x)+f(y)+2 B(x, y)$.

Using (15) we arrive at

$2 d_{1}\left(D_{2}(x, y)\right)+D_{1}\left(d_{1}(x), d_{1}(y)\right)+2 D_{1}\left(d_{2}(x), D_{2}(x, y)\right)+2 D_{1}\left(d_{2}(y), D_{2}(x, y)\right)=B(x, y)$.

Substituting in the equation above $x$ by $-x$ we obtain by comparing this new equation with the equation above that

$$
2 D_{1}\left(d_{2}(x), D_{2}(x, y)\right)+2 D_{1}\left(d_{2}(y), D_{2}(x, y)\right)=B(x, y) \text { for all } x, y \in M .
$$

Let us replace in (16) $x$ by $2 x$. We have

$$
8 D_{1}\left(d_{2}(x), D_{2}(x, y)\right)+2 D_{1}\left(d_{2}(y), D_{2}(x, y)\right)=B(x, y) \text { for all } x, y \in M \text {. }
$$


By comparing (16) and (17) we obtain

$$
\begin{aligned}
& 6 D_{1}\left(d_{2}(x), D_{2}(x, y)\right)=0 \\
& \Rightarrow D_{1}\left(d_{2}(x), D_{2}(x, y)\right)=0 \text { for all } x, y \in M .
\end{aligned}
$$

since $M$ is 2 and 3-torsion free. From (18) it follows that both terms on the left side of the relation (16) are zero, which means that $B=0$. Hence (15) reduces to

$$
d_{1}\left(d_{2}(x)\right)=0 \text { for all } x \in M .
$$

Let in (18) $y$ be $y \alpha x$. We have

$$
\begin{aligned}
0 & =D_{1}\left(d_{2}(x), D_{2}(x, y \alpha x)\right) \\
& =D_{1}\left(d_{2}(x), D_{2}(x, y) \alpha x+y \alpha d_{2}(x)\right) \\
& =D_{1}\left(d_{2}(x), D_{2}(x, y) \alpha x\right)+D_{1}\left(d_{2}(x), y \alpha d_{2}(x)\right) \\
& =D_{1}\left(d_{2}(x), D_{2}(x, y)\right) \alpha x+D_{2}(x, y) \alpha D_{1}\left(d_{2}(x), x\right)+D_{1}\left(d_{2}(x), y\right) \alpha d_{2}(x)+y \alpha d_{1}\left(d_{2}(x)\right)
\end{aligned}
$$

for all $x, y \in M, \alpha \in \Gamma$.

This implies

$D_{1}\left(d_{2}(x), y\right) \alpha d_{2}(x)+D_{2}(x, y) \alpha D_{1}\left(d_{2}(x), x\right)=0$ for all $x, y \in M, \alpha \in \Gamma$.

according to (18) and (19). Let us replace in (20) $y$ by $x \beta y$. We have

$$
\begin{aligned}
0= & D_{1}\left(d_{2}(x), x \beta y\right) \alpha d_{2}(x)+D_{2}(x, x \beta y) D_{1}\left(d_{2}(x), x\right) \\
= & D_{1}\left(d_{2}(x), x\right) \alpha y \beta d_{2}(x)+x \beta D_{1}\left(d_{2}(x), y\right) \alpha d_{2}(x)+d_{2}(x) \alpha y \beta D_{1}\left(d_{2}(x), x\right) \\
& +x \alpha D_{2}(x, y) \beta D_{1}\left(d_{2}(x), x\right) \\
= & D_{1}\left(d_{2}(x), x\right) \alpha y \beta d_{2}(x)+d_{2}(x) \alpha y \beta D_{1}\left(d_{2}(x), x\right)+x \beta\left(D_{1}\left(d_{2}(x), y\right) \alpha d_{2}(x)\right. \\
& \left.+D_{2}(x, y) \alpha D_{1}\left(d_{2}(x), x\right)\right) \text { for all } x, y \in M, \alpha, \beta \in \Gamma .
\end{aligned}
$$

Now, by (20), we arrive finally at

$$
D_{1}\left(d_{2}(x), x\right) \alpha y \beta d_{2}(x)+d_{2}(x) \alpha y \beta D_{1}\left(d_{2}(x), x\right)=0 \text { for all } x, y \in M, \alpha, \beta \in \Gamma .
$$

From the relation above one can conclude that $D_{1}\left(d_{2}(x), x\right)=0$ is fulfilled for all $x \in M$. Namely, if $D_{1}\left(d_{2}(x), x\right) \neq 0$ for some $x \in M$, then $d_{2}(x)=0$ according to (21) and Lemma 2.2, contrary to the assumption $D_{1}\left(d_{2}(x), x\right) \neq 0$. Therefore, since $D_{1}\left(d_{2}(x), x\right)=0$ for all $x \in M$, the proof of the theorem is complete since all the requirements of Theorem 2.5 are fulfilled.

In case $D_{1}=D_{2}$ Theorem 2.7 can be proved for semi-prime $\Gamma$-rings.

Theorem 2.8. Let $M$ be a 2, 3-torsion free semiprime $\Gamma$-ring satisfying the condition (*). Let $D: M \times M \rightarrow M$ and $B: M \times M \rightarrow M$ be a symmetric bi-derivation and a symmetric bi-additive mapping, respectively. Suppose that $d(d(x))=f(x)$ holds for all $x \in M$, where $\mathrm{d}$ is the trace of $D$ and $f$ is the trace of $B$. Then $D=0$. 
Proof. Obviously, we can use the beginning of the proof of Theorem 2.5. In this case relations (18) and (19) can be written in the form

$$
D(d(x), D(x, y))=0 \text { for all } x, y \in M \text {. }
$$

and

$$
d(d(x))=0 \text { for all } x \in M
$$

Let us write in (22) $y \alpha z$ instead of $y$. We have

$$
\begin{aligned}
0= & D(d(x), D(x, y \alpha z)) \\
= & D(d(x), D(x, y) \alpha z+y \alpha D(x, z)) \\
= & D(d(x), D(x, y) \alpha z)+D(d(x), y \alpha D(x, z)) \\
= & D(d(x), D(x, y)) \alpha z+D(x, y) \alpha D(d(x), z)+D(d(x), y) \alpha D(x, z)+y \alpha D(d(x), D(x, z)) \text { for } \\
& \text { all } x, y, z \in M, \alpha \in \Gamma .
\end{aligned}
$$

Hence by (22) we have

$$
D(x, y) \alpha D(d(x), z)+D(d(x), y) \alpha D(x, z)=0
$$

and, in particular, for $z=d(x)$ we obtain

$$
D(d(x), y) \alpha D(x, d(x))=0 \text { for all } x, y \in M, \alpha \in \Gamma .
$$

according to (23). Replace in (24) $y$ by $x \beta y$. We have $0=D(d(x), x \beta y) \alpha D(x, d(x))=$ $D(d(x), x) \beta y \alpha D(x, d(x))+x \beta D(d(x), y) \alpha D(x, d(x))$ which leads to

$D(d(x), x) \alpha y \beta D(d(x), x)=0 ; x, y \in M, \alpha, \beta \in \Gamma$; and we obtain $D(d(x), x)=0$ for all $x \in M$ by the semiprimeness of M. Thus by Theorem 2.6 the proof is complete.

Acknowledgement. The authors would like to express their gratitude to the referee for valuable suggestions and helpful comments.

\section{REFERENCES}

[1] Barnes, W. E., On the Г-rings of Nobusawa, Pacific J. Math., 18 (1966), 411- 422.

[2] Dey, K. K., Paul, A. C. and Rakhimov, I. S., On Commutativity in Gamma-near-ring with Symmetric Bi- $(\sigma, \tau)$-derivations, Far East Journal of Mathematical Sciences, 65(2) (2012), 211-225.

[3] Maksa, G., A remark on symmetric bi-additive functions having nonnegative diagonalization, Glasnik Mat., 15(35) (1980), 279-282.

[4] Maksa, G., On the trace of symmetric bi-derivations. C. R. Math. Rep. Acad. Sci. Canada, 9(6) 1987, 303-307.

[5] Nabusawa, N., On a generalization of the Ring Theory, Osaka J. Math., 1, (1964), 65-75.

[6] Ozturk, M. A., Sapanci, M., On generalized symmetric bi-derivations in prime ring, East Asian Math. J., 15(2) (1999), 165-176.

[7] Ozturk, M. A., Sapanci, M., Soyturk, M. and Kim, K. H., Symmetric bi-derivations on prime gamma ring, Scientiae Math., 3(2) (2000), 273- 281.

[8] Posner, E., Derivations in Prime Rings. Proc. Amer. Math. Soc. 8 (1957), 1093-1100.

[9] Sapanci, M., Ozturk, M.A. and Jun, Y. B., Symmetric bi-derivations on prime rings, East Asian Math. J., 15(1) (1999), 105-109.

[10] Vukman, J., Symmetric bi-derivation on prime and semiprime rings, Aequations Mathematicae, 38 (1989) 245-254. 